\title{
Hidden Markov Model Analysis for Space Shuttle Crewmembers' Scanning Behavior
}

\author{
Miwa Hayashi \\ San Jose State University Foundation \\ NASA Ames Research Center \\ Moffett Field, CA, USA \\ mhayashi@mail.arc.nasa.gov
}

\author{
Brent Beutter \\ Robert S. McCann \\ Human Factors Research and Technology Division \\ NASA Ames Research Center \\ Moffett Field, CA, USA \\ \{Brent.R.Beutter, Robert.S.McCann\}@nasa.gov
}

\begin{abstract}
The paper describes the application of a novel analysis technique employing Hidden Markov Models (HMMs) to analyze complex sequences of visual fixations and understand the supervisory monitoring strategies of Space Shuttle cockpit crewmembers. While HMM analysis has been shown to be useful for understanding the scanning behavior of aircraft pilots engaging in manual flight control, it has not yet been applied to the supervisory monitoring context. The paper presents a proof-of-concept demonstration by applying HMM analysis to the eyemovement data of a veteran Commander astronaut performing simulated ascent-phase operations. The paper also demonstrates how the resulting HMM parameters can be used to detect deviations from regular scan patterns.
\end{abstract}

Keywords: Automation, eye movements, Hidden Markov Model (HMM), saccades, scan patterns, Space Shuttle cockpit displays, supervisory monitoring, visual search.

\section{Introduction}

Operating the Space Shuttle is a complex task; a single vehicle functions as a launch rocket during ascent, an orbital spacecraft during orbit, and a hypersonic plane and subsonic glider during entry and landing. The ascent and entry phases are especially critical, and the cockpit crewmembers must closely monitor a large amount of system-status and navigational information presented on various cockpit displays during these phases. Understanding how well-trained crewmembers manage to efficiently scan critical information during the busiest phases of flight is of great interest to NASA human-factors researchers. Such knowledge will help improve existing astronaut training programs, and also inform the design of more human-centered cockpit display systems for nextgeneration spacecraft.

This paper proposes a novel analysis tool based on Hidden Markov Models (HMMs) to analyze astronaut supervisory monitoring behavior. The basic assumption of our HMM analysis is that crewmembers' eye movements (the observation-symbol process) are driven by their internal cognitive state, i.e., the specific monitoring task in which the crewmember is engaged (the hidden-state process). The transitions among the monitoring tasks are assumed to follow first-order Markov-process transition rules, and the probability distributions of the crewmembers' eye movements are assumed to depend on which monitoring task is being performed. The two-layered structure of HMMs allows the probabilistic characteristics of the scan patterns to dynamically change during flight. The inherent flexibility of HMM analysis is a significant advantage over other conventional analysis approaches, which implicitly assume that the scanning process is statistically time-invariant (e.g., calculating simple eyemovement statistics, such as mean fixation durations or frequencies over specific time intervals). In addition, HMM analysis hypothesizes that the dependency between the internal cognitive process and the observed eye movements is stochastic, rather than deterministic. This stochastic dependency provides robustness in applying the model to the noisy or ambiguous real-world data.

HMM analysis has been shown to be useful in understanding aircraft pilots' scanning behavior while manually flying instrument approaches [1]. These pilots were performing concurrent manual tracking tasks along multiple axes (i.e., vertical, horizontal, and airspeed axes). Pilots are usually taught to frequently crosscheck the instruments related to the same tracking axis (e.g., pitch, altitude, and glide-slope deviations for vertical-axis tracking). Also the aircraft dynamics, the bandwidth of the pilot's inputs, and the bandwidth of disturbance along each axis determine how often each set of instruments should be scanned [2]. These factors function as a set of external constraints on the pilot's scanning behavior while manually controlling a flight. In contrast, with the exception of a

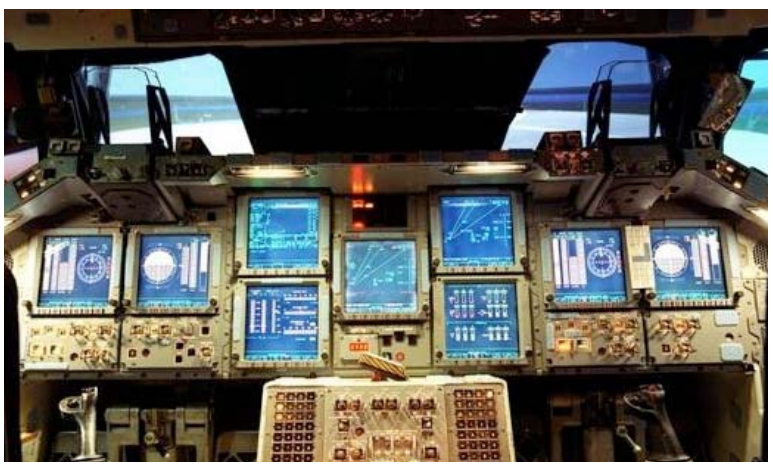

Figure 1.Space Shuttle MEDS Cockpit (NASA photo) 
short period of descent prior to touchdown, the tasks that the Shuttle crewmembers perform are mostly supervisory monitoring of heavily automated processes. Not having to manually control the vehicle gives the crewmembers considerable liberty in the way they scan the instruments. This condition poses additional challenges to the researcher, as fewer assumptions about scanning behavior can be made for setting the initial model structure. However, we demonstrate that HMM analysis can be successfully applied to the scanning behavior of the crewmembers (with some modifications), and can help in understanding their supervisory monitoring strategies.

The paper presents a proof-of-concept demonstration of HMM analysis of the eye-movement data of an experienced Commander astronaut collected during parttask simulations of nominal Shuttle ascent. Selection of the hidden states, estimation of the model parameters, and cross-validation of the estimated model parameters are demonstrated and discussed. The paper also demonstrates the use of estimated HMM parameters to identify the crewmember's temporary deviation from regular scan patterns. Finally, the potential advantages and limitations of HMM analysis are discussed.

\subsection{Monitoring Tasks During Ascent}

We begin by providing an outline of the Shuttle cockpit display systems and the monitoring tasks that crewmembers perform during the ascent phase of flight. Figure 1 shows the Shuttle glass cockpit called Multifunction Electronic Display System (MEDS). The MEDS cockpit seats two crewmembers: the Commander in the left seat and the Pilot in the right seat. Figure 2 illustrates the Commander-side display arrangement in the MEDS cockpit used in the simulations, along with the display name acronyms used in this paper. The HSI/SPD (2) and the ADI/ALT (3) are flight displays similar to those used in commercial air transporter cockpits. The F2-F3 and F6 BTM are switch panels (F2, F3, and F6 are the names of the corresponding forward switch panels). The rest of the displays (5-12) are shared with the Pilot astronaut in the
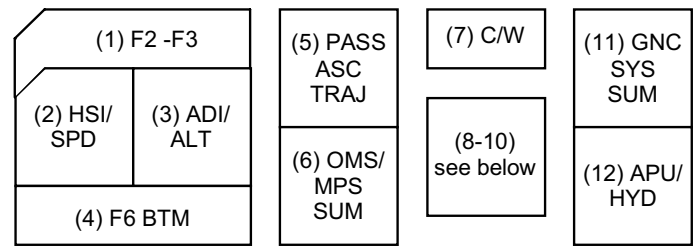

(1) F2-F3: Switch panels including Digital Autopilot (DAP) switches

(2) Horizontal Situation Indicator (HSI) / Speed indicators (SPD)

(3) Attitude Director Indicator (ADI) / Altitude Indicators (ALT)

(4) F6 Bottom (BTM): Switch panel including Attitude switch

(5) Primary Avionics Software System (PASS) Ascent Trajectory (ASC TRAJ)

(6) Orbital Maneuvering System (OMS) / Main Propulsion System (MPS) Summary (SUM)

(7) Caution \& Warning Matrix (C/W)

(8) Backup Flight System (BFS) ASC TRAJ

(9) BFS System (SYS) SUM 1

(10) Fault Log

(11) Guidance Navigation Control (GNC) SYS SUM

(12) Auxiliary Power Unit (APU) / Hydraulics (HYD) SUM

Figure 2. Display Arrangement for Commander Side: Ascent Configuration

right seat. The three displays (8-10) share one display area, and are toggled by keyboard inputs. When neither the BFS ASC TRAJ (8) nor the Fault Log (10) is being used, the crewmembers usually have the systems status, the BFS SYS SUM 1 (9), up on this display.

The ascent phase includes several critical events that crewmembers must monitor at specific times. Table 1 summarizes these critical events and the associated information that the crewmembers are supposed to monitor, as listed in the ascent checklists [3].

When not monitoring critical events listed in Table 1, the crewmembers scan the displays overall so that they remain apprised of system health as well as maintain situation awareness. This regular scanning is an important task; yet, unlike the critical-event monitoring, there is no written protocol describing how this regular scan should be performed. Hence, the regular scan patterns were derived directly from the actual eye-movement data.

Table 1. Critical-event monitoring tasks during the ascent phase

\begin{tabular}{|c|c|c|c|}
\hline $\begin{array}{l}\text { Approx. Mission } \\
\text { Elapsed Time } \\
\text { (MET) [min:sec] }\end{array}$ & Critical Events & What to Monitor & $\begin{array}{l}\text { Display(s) to be Monitored } \\
\text { (see Figure 2) }\end{array}$ \\
\hline 0:01 & Launch & Digital Autopilot (DAP) Pitch and Roll/YAW switches are still in AUTO. & F2-F3 \\
\hline 0:07 & Roll Program & $\begin{array}{l}\text { Vehicle rolls to heads-down ascent attitude. Move Attitude switch to } \\
\text { LVLH (Local Vertical Local Horizontal) position. }\end{array}$ & ADI/ALT, BFS ASC TRAJ, F6 BTM \\
\hline $0: 30-1: 00$ & Thrust Bucket & $\begin{array}{l}\text { MPS throttling reduces to } 67 \% \text { to limit max flight dynamic pressure, then } \\
30 \text { seconds later, comes back to } 104 \% \text {. }\end{array}$ & OMS/MPS SUM \\
\hline Around 2:00 & $\begin{array}{l}\text { Solid Rocket } \\
\text { Booster (SRB) } \\
\text { Separation }\end{array}$ & $\begin{array}{l}\text { (i) Pressure level inside SRB chamber falls below } 50 \text { psi, which triggers } \\
\text { the SRB separation. (ii) Major Mode (MM) changes from } 102 \text { to } 103 \text {. (iii) } \\
\text { Check that Time to MECO (TMECO) indications on the PASS and BFS } \\
\text { ASC TRAJ displays converge. }\end{array}$ & (i)-(iii) PASS \& BFS ASC TRAJ \\
\hline Around 3:00 & --- & Check the flash evaporator temperature is $<60^{\circ} \mathrm{F}$. & Overhead panel \\
\hline $5: 40$ & $\begin{array}{l}\text { Roll to } \\
\text { Heads Up }\end{array}$ & Vehicle rolls to heads-up attitude. & ADI/ALT \\
\hline $8: 30$ & $\begin{array}{l}\text { Main Engine } \\
\text { Cutoff } \\
\text { (MECO) }\end{array}$ & $\begin{array}{l}\text { (i) MPS thrust level drops to } 0 \% \text {. (ii) MAIN ENGINE STATUS lights } \\
\text { illuminate red. (iii) Cutoff bug on BFS ASC TRAJ indicates MECO } \\
\text { velocity. }\end{array}$ & $\begin{array}{c}\text { i) OMS/MPS SUM, ii) MAIN } \\
\text { ENGINE STAUS lights (located } \\
\text { below BFS ASC TRAJ), iii) BFS } \\
\text { ASC TRAJ }\end{array}$ \\
\hline
\end{tabular}




\subsection{Hidden Markov Model (HMM) Analysis}

A brief overview of HMMs follows. (For complete descriptions of the HMM algorithms, see [4].) The structure of an HMM consists of two layers of stochastic processes. The hidden-state process is not directly observable and follows first-order Markov process transition rules. The observation-symbol process is a physically observable process, and has probability distributions that depend on the current hidden state. An HMM model structure is completely described by a set of three model-parameter matrices, $\lambda=(A, B, \pi)$. The statetransition probability distribution matrix, $A(i, j)(1 \leq i, j \leq$ $N)$, defines the transition probability from the hidden state, $i$, to the hidden state, $j$. The observation symbol probability distribution matrix, $B(j, k)(1 \leq j \leq N, 1 \leq k \leq M)$, defines the probability of the observation symbol, $v_{k}$, being observed given hidden state, $j$. The initial state probability distribution matrix, $\pi(i)(1 \leq i \leq N)$, defines the probability that the hidden state, $i$, is the initial state. Given an observation-symbol sequence, $O=\left\{o_{1}, \ldots, o_{T}\right\}$, and a set of initial conditions of the model parameter matrices, $\lambda_{0}=\left(A_{0}\right.$, $\left.B_{0}, \pi_{0}\right)$, the HMM algorithms iteratively calculate the most likely sequence of the hidden states, $q^{*}=\left\{q_{1}, \ldots, q_{T}\right\}$, and re-estimate the model parameters, $\lambda$.

In this HMM analysis, the observation-symbol sequence corresponds to the crewmember's display fixation sequence, and the hidden states to the critical-event monitoring tasks (listed in Table 1) and the regularscanning tasks (determined from the actual eye-movement data). Figure 3 illustrates the concept of this HMM structure. The diagram in Figure 3 includes the $N_{c}$ criticalevent hidden states (the circles denoted $C_{l}, \ldots, C_{N c}$ ) and the $N_{r}$ regular-scanning hidden states (the circles denoted $R_{l}$, $\ldots, R_{N r}$; Note $N_{c}+N_{r}=N$ ). Each hidden state has its own fixation probability distributions. The arrows indicate hidden-state transitions, including recurring transitions to the same hidden state. (To avoid cluttering the diagram, the two arrows between an individual critical-event hidden state and the box around the regular-scanning hidden state represent the transitions between this specific critical-event hidden state and any of the regular-scanning hidden states.)

The diagram in Figure 3 indicates no direct transition within the critical-event hidden states. This prevents two critical-event hidden states from occurring successively. The inhibition of the transitions between any of the two critical-event hidden states was implemented as follows: First, let the first $N_{c}$ hidden states, $i_{l}, \ldots, i_{N c}$, be the criticalevent hidden states, and the remaining $N_{r}$ hidden states, $i_{N C+1}, \ldots, i_{N}$, be the regular-scanning hidden states. Then, the model-parameter matrices could be partitioned into submatrices as in the following equations. The subscript, $c$, denotes the critical-event hidden-state class, and the subscript, $r$, the regular-scanning hidden-state class:

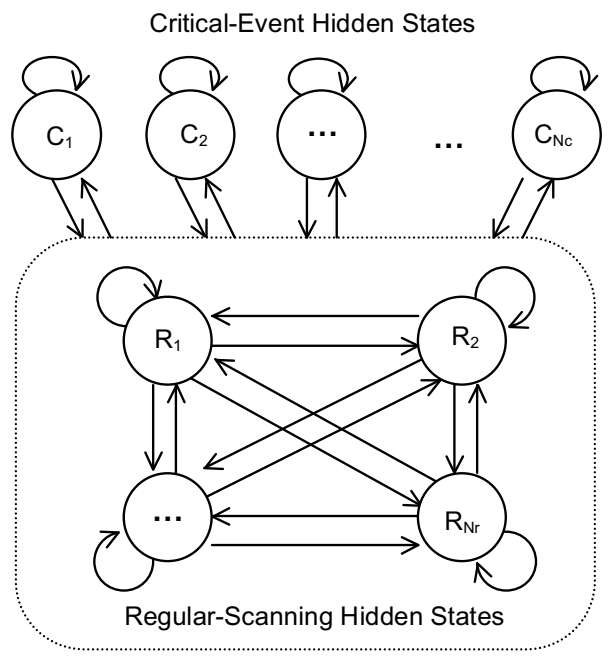

Figure 3. HMM structure for crewmembers' scanning behavior.

$$
\begin{aligned}
& A=\left[\begin{array}{ll}
A_{c c} & A_{c r} \\
A_{r c} & A_{r r}
\end{array}\right] \\
& B=\left[\begin{array}{l}
B_{c} \\
B_{r}
\end{array}\right] \\
& \pi=\left[\begin{array}{ll}
\pi_{c} & \pi_{r}
\end{array}\right]
\end{aligned}
$$

where $A_{x y}\left(x, y \in\{c, r\}, N_{x} \times N_{y}\right)$ is the hidden-state transition probabilities from each hidden state of the class, $x$, to each hidden state of the class, $y ; B_{x}\left(N_{x} \times M\right)$ is the observation-symbol probabilities given each hidden state of the class, $x$; and $\pi_{x}\left(1 \times N_{x}\right)$ is the initial state probabilities of each hidden state of the class, $x$. Then, the transitions between two critical-event hidden states can be prohibited by setting the sub-matrix, $A_{c c}$, a pure diagonal matrix.

The elements of the other sub-matrices of $A$ (i.e., $A_{c r}$, $A_{r c}$, and $A_{r r}$ ) generally remain non-zero to allow the corresponding hidden-state transitions. However, the nonzero elements of the $A_{r c}$ sub-matrix may cause problems in the estimation of the critical-event hidden states. These hidden states were supposed to occur only near the time at which the corresponding critical event occurs. Yet, because of the non-zero $A_{r c}$, the HMM may estimate that the critical-event hidden state occurred at other times due to the occurrence of scan patterns that happened to be similar to it. To prevent this type of estimation error, some critical-event hidden states were restricted to occur only at specific times. For instance, the Thrust Bucket critical-event hidden state was associated with the OMS/MPS SUM (6) fixations that occurred only between 0:30 1:00 MET. (This was easily implemented by adding a new column to the $B$ matrix, representing the OMS/MPS SUM (6) fixations observed 
specifically during 0:30 1:00 MET.) Similarly, BFS ASC TRAJ (8) associations to the hidden states were also time dependent. Those occurring near 0:07 MET were associated with the Roll Program hidden state, those around 2:00 MET with the SRB Separation hidden state, and those around 8:30 MET with the MECO hidden state. The BFS ASC TRAJ (8) fixations that occurred at other times were associated with some of the regular-scanning hidden states.

As mentioned previously, regular scanning is an important task, and researchers may wish to know when and how long the crewmember's scan patterns deviated from it. Such information may help, for instance, in training astronauts to maintain a proper scan, or in developing a cockpit display system that facilitates the crewmembers' regular scanning. The HMM sub-model parameters, $\lambda_{r}=\left(A_{r r}, B_{r}, \pi_{r}\right)$, represent a reduced HMM structure that specifies how fixations occur during regular scanning. (The elements of the $A_{r r}$ and $\pi_{r}$ matrices must be re-normalized so that $\Sigma_{j}\left[A_{r r}(i, j)\right]=1$, and $\Sigma_{i}\left[\pi_{r}(i)\right]=1$.) This reduced HMM structure can be used to detect deviations of the crewmember's fixation sequence from the nominal scan patterns. The Viterbi algorithm [4] is a dynamic programming algorithm that computes the most likely sequence of the hidden states given a particular observation sequence, $O$, and particular HMM model parameters, $\lambda$. During this computation process, the algorithm also computes the probability, $P^{*}$, of observing that fixation sequence given those model parameters, which could be useful for our purpose. (The $P^{*}$ values were also used in the cross-validation process of the estimated model parameters-see Section 3.2.) To detect deviations from the nominal scan patterns, the most recent $\tau$ consecutive fixation data from each time point were subjected to the Viterbi algorithm with the reduced model parameters, $\lambda_{r}$, to compute continuous $P^{*}(t)$. When the most recent $\tau$ fixation data points indicated deviation from the nominal scan patterns, the Viterbi algorithm returned lower $P^{*}$ values.

\section{Simulation}

A fixed-base, part-task Shuttle cockpit simulator at NASA Ames Research Center was used to conduct the ascent-phase operation simulation and collect eyemovement data. The simulator was configured to replicate the Commander-side seat environment of the Shuttle MEDS cockpit. Four 20" touch-screen LCD monitors in front of the operator provided eight displays on the front panel as shown in Figure 2. Two 20" touch-screen LCD monitors on the left, three on the right, and two overhead were used to simulate switch panels. To the right of the participant's right knee, a 12" touch-screen LCD monitor was placed to simulate a keyboard. A network of seven PCs and an SGI Octane was used to compute the Shuttle's flight dynamics and the display output. The flight dynamics, system parameter tables, and engine sound were obtained through NASA Johnson Space Center. The display graphics were generated using VAPS, OpenGL ${ }^{\circledR}$, and winGDI.

The simulation included operations from launch to MECO, approximately 8.5 minutes in duration. Within the four trials conducted, the first and fourth were nominal trials, and the second and third were off-nominal trials, which contained some simulated system malfunctions. The data from the two nominal trials were used to estimate the HMM model parameters. The data from the third trial (offnominal) were used for identification of the participant's deviation from the regular scan patterns. The system malfunction inserted in the third trial was a low ullage pressure condition in the liquid hydrogen external storage tank, which occurred at 1:55 $(115 \mathrm{sec})$ MET. The data from the second trial were not used in this paper.

The participant was a veteran Commander astronaut. Before data collection trials started, the participant performed practice trials in the simulator. During the data collection trials, the participant's eye-movement data (i.e., lines-of-sight data) were collected at $60 \mathrm{~Hz}$ with a headmounted eye camera (ISCAN ETL-500, ISAN, Inc., Burlington, MA) and head tracker (FasTRAK, Polhemus, Colchester, VT). The participant's switch-throw activities were also recorded. Each trial was also videotaped.

The intersections of the participant's lines-of-sight and the display surfaces were computed from the data. Then, saccades were eliminated by omitting points where the running averages of the intersections failed to meet our fixation criteria (i.e., staying within a circle of a 3/4-inch radius on the monitor surface for at least $150 \mathrm{msec}$ ). Each remaining saccade-free data point was assigned to a display area based on its location, and if the duration within a display area was less than 0.5 seconds, it was counted as a single fixation. Longer durations were assigned to be multiple consecutive fixations at the same display area, by limiting the maximum duration of each additional fixation to be 0.5 seconds (e.g., if a duration was 0.3 seconds, it was counted as one fixation; if 0.6 seconds, two fixations; if 1.2 seconds, three fixations; etc.). The resulting sequences of fixations were used for the display fixation transition probability computations and the HMM analysis.

\section{Results}

\subsection{Selection of Hidden States}

While the critical-event hidden states were selected based on the ascent checklists, the regular-scanning hidden states had to be derived from the actual eye-movement data. The fixation transition probabilities among displays were computed to identify patterns in the regular scanning and select regular-scanning hidden states.

Analysis revealed very frequent eye traffic between the HSI/SPD (2) and the ADI/ALT (3) displays (transition 
probabilities given the origin displays: $49.2 \%$ for the HSI/SPD (2) to the ADI/ALT (3); $58.4 \%$ for the opposite direction). Both of these displays provide primary flight instrument information. Thus, in the following HMM analysis, the pair of these displays constitutes the Flight Instruments hidden state.

Relatively heavy eye traffic was also observed between the OMS/MPS SUM (6) and the BFS SYS SUM 1 (9) $(29.9 \%$ for the OMS/MPS SUM (6) to the BFS SYS SUM 1 (9); 35.3\% for the opposite direction). Both include system information, and, thus, these displays were assigned to be the System hidden state. In addition, this System hidden state was also chosen to include other systeminformation displays, which were fixated much less frequently: the C/W (7), the GNC SYS SUM 1 (11), the APU/HYD (12), the overhead panel, and the left and right switch panels.

The analysis also indicated relatively large transition probabilities to the PASS ASC TRAJ (5) from all the major displays (i.e., the HSI/SPD (2), the ADI/ALT (3), the OMS/MPS SUM (6), and the BFS SYS SUM 1 (9) (ranged between 18.4 27.7\%)). The PASS ASC TRAJ (5) also showed almost uniformly distributed transition probabilities to all of these major displays (ranged between 17.0 24.4\%). This pattern strongly indicates that the PASS ASC TRAJ (5) worked like a hub. The major pieces of information the PASS ASC TRAJ (5) presents are Time to Main Engine Cutoff (TMECO), the abort options, the MET, and the Major Mode (MM), which are all related to overall mission situation awareness (SA). Therefore, the PASS ASC TRAJ (5) display was assigned to the Mission SA hidden state.

Occasionally, the participant fixated on the F2-F3 (1) during flight, presumably to check the Digital Autopilot (DAP) status. Thus, a DAP check hidden state was also included in the regular-scanning hidden states. Finally, to make the list complete, a BFS Traj/Fault Log Check hidden state was added to include fixations of the BFS ASC TRAJ (8) and the Fault $\log (10)$, the displays only rarely fixated during regular scanning.

Figure 4 illustrates the HMM structure derived from the fixation transition probability analyses described above. Table 2 summarizes observations associated with each hidden state in Figure 4. In Figure 4, the three major regular-scanning hidden states (i.e., Flight Instruments, Mission SA, and System) are depicted as shaded circles with thick transition arrows. These three major hidden states occurred much more frequently than the remaining two regular-scanning hidden states, and thus were dominant during the regular scanning process. Note that some critical-event hidden states were omitted to improve computation efficiency. The Launch critical event hidden state was not included in this HMM structure; the DAP check required at launch was detected as a part of the DAP-

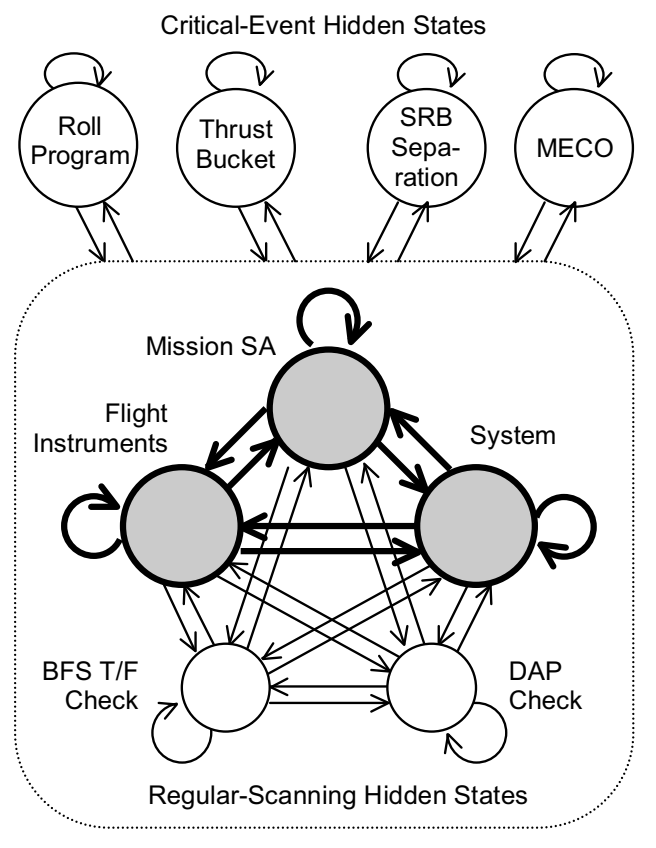

Figure 4. HMM structure for the participant's scanning behavior

Table 2. HMM structure details

\begin{tabular}{|c|c|c|}
\hline Hidden States & Class & Observations \\
\hline Roll Program & c & $\begin{array}{l}\text { ADI, BFS ASC TRAJ, F6 BTM (MET < } \\
0: 20)\end{array}$ \\
\hline Thrust Bucket & c & OMS/MPS $(0: 30 \leq$ MET < 1:00) \\
\hline $\begin{array}{c}\text { SRB } \\
\text { Separation }\end{array}$ & c & $\begin{array}{l}\text { PASS ASC TRAJ, BFS ASC TRAJ (MET } \\
\text { 2:00) }\end{array}$ \\
\hline MECO & c & $\begin{array}{l}\text { OMS/MPS SUM, BFS ASC TRAJ (MET } \\
8: 30), \text { HSI/SPD }\end{array}$ \\
\hline $\begin{array}{c}\text { Flight } \\
\text { Instruments }\end{array}$ & $r$ & $\begin{array}{l}\text { F2-F3, HSI/SPD, ADI/ALT, F6 BTM (MET } \geq \\
0: 20)\end{array}$ \\
\hline Mission SA & $\mathrm{r}$ & PASS ASC TRAJ \\
\hline System & $r$ & $\begin{array}{l}\text { OMS/MPS (MET < 0:30 or MET } \geq 1: 00 \text { ), } \\
\text { C/W, BFS SYS SUM, GNC SYS SUM, } \\
\text { APU/HYD, overhead switch panel, left } \\
\text { switch panel, right switch panel }\end{array}$ \\
\hline $\begin{array}{l}\text { BFS Traj/Fault } \\
\text { Log Check }\end{array}$ & $r$ & $\begin{array}{l}\text { BFS ASC TRAJ (MET not around the SRB } \\
\text { Separation or MECO), Fault Log }\end{array}$ \\
\hline DAP Check & $r$ & F2-F3 \\
\hline
\end{tabular}

Check regular-scanning hidden state, instead. Also, criticalevent hidden states for the flash evaporator temperature check at 3:00 MET and for the ADI check for the roll-toheads-up at 5:40 MET were not included, because these fixations do not yield distinguishable scan patterns, such as frequent crosscheck with other displays or extremely long fixation durations on specific displays. Instead, these scanning behaviors were identified as a part of the System or Flight Instrument regular-scanning hidden states.

Also notice that the Flight Instruments hidden state in Table 2 includes the F2-F3 (1) in addition to the HSI/SPD (2), the ADI/ALT (3), and the F6 BTM (after 0:20 MET). This was done because the F2-F3 (1) switch panel was located very close to the HSI/SPD (2) and the ADI/ALT (3), and, therefore, some of the fixation points on the HSI/SPD (2) or the ADI/ALT (3) could be accidentally classified into 
the adjacent F2-F3 (1) area. However, by allowing the data within the adjacent area to be accounted for by the Flight Instruments hidden state with a relatively smaller weight (i.e., the corresponding element in the $B$ matrix), the effects of the misclassifications can be reduced. This softbordering capability is one of the advantages of HMM analysis and will be discussed later.

\subsection{HMM Analysis Results}

Based on the above-mentioned HMM structure, the HMM algorithms [4] were run to estimate the maximumlikelihood model parameters given the participant's fixation sequence data. Two sets of HMM parameters were estimated from the fixation data collected during two nominal trials: $\lambda_{\# 1}$ was estimated with the fixation sequence from trial $\# 1$, and $\lambda_{\# 4}$ with the sequence from trial \#4. To determine which set of the model parameters represents the participant's scanning behavior better, a cross-validation was performed by computing the degree to which $\lambda_{\# l}$ could describe the fixation sequence of trial \#4 and the degree to which $\lambda_{\# 4}$ could describe the fixation sequence of trial \#1. The Viterbi algorithm was used to compute the probability, $P^{*}$, of the observed fixation sequence assuming the given model parameters. In this cross-validation, $\lambda_{\# 1}$ yielded higher $P^{*}$ with the trial \#4 fixation data than $\lambda_{\# 4}$ did with the trial \#1 fixation data. Thus, the model parameters, $\lambda_{\# 1}$, were considered a better description of the participant's data than $\lambda_{\# 4}$, and $\lambda_{\# 1}$ is used in the following demonstrations. Figure 5 shows time plots of the fixation sequence from trial \#4 along with the hidden-state sequence estimated using the model parameters, $\lambda_{\# 1}$.

\subsection{Deviations from Regular Scanning}

From $\lambda_{\# 1}$, a reduced set of model parameters, $\lambda_{r}=\left(A_{r r}\right.$, $\left.B_{r}, \pi_{r}\right)$, which included only the regular-scanning hidden states, was extracted. Then, again, the Viterbi algorithm was applied to $\lambda_{r}$ and the last $\tau$ consecutive fixation data points from each time point to compute $P^{*}(t) \quad(\tau=30$, approximately equivalent to 15 seconds, was used in this study). As mentioned previously, if the Viterbi algorithm returns low values of the probability, $P^{*}(t)$, at time, $t_{l}$, that indicates that the $\tau$ fixation data points immediately prior to the time point, $t_{1}$, could not be described well by the given HMM structure containing only the regular-scanning hidden states. Figure 6 shows time plots of the logarithms of the resulting probabilities, $\log P^{*}(t)$. (Natural logarithms of $P^{*}(t)$ were taken because the values of $P^{*}(t)$ were very small.) The top and middle plots were computed with the fixation sequence from the nominal trials $\# 1$ and \#4, respectively. Indeed, the plots show clear reductions of $\log$ $P^{*}(t)$ around the critical event times, where the scanning behavior should deviate from the regular-scanning patterns.

An analogous computation with the same model parameters, $\lambda_{r}$, was applied to the fixation sequence from the off-nominal trial \#3, where a system malfunction occurred at 1:55 (115 seconds) MET. This result is plotted at the bottom of Figure 6. The plot indicates that the participant deviated from the regular scan patterns a couple of times after the malfunction occurrence. Actually, the participant correctly completed the malfunction management procedure by 2:35 (155 sec) MET, but, even after that, still kept staring at the right panel for a long time. (The voice recording on the videotape indicated that the participant was trying to make sure all the switch positions on the right panel were correct.) Therefore, the first dip in Figure 6, around $150 \mathrm{sec}$ MET, was for correcting the malfunction, and the following two dips (around $215 \mathrm{sec}$ and $270 \mathrm{sec}$ MET) were caused by the participant staring at the right panel.

\section{Discussion}

In this proof-of-concept study, an HMM structure for the participant's supervisory monitoring scanning behavior was derived. The major differences of this HMM structure from those in the aircraft pilots' scanning behavior study in [1] were that the hidden states had two classes-criticalevent monitoring and regular scanning - and that a portion of the $A$ matrix and the set of the observation symbols were modified to reduce the estimation errors of the timespecific critical-event hidden states. Due to the lack of formal validation, the results of the present study should be considered preliminary. However, the original purpose of this study was to demonstrate a proof of concept and to illustrate potential advantages and limitations of HMM analysis for future use, and the study successfully met these intended goals. We now turn to a discussion of some of the advantages and limitations of the HMM analysis approach.

One of the major advantages of HMM analysis, as pointed out in [1], arises when information sampling from a single display can be associated with more than one hidden state. For instance, the ADI was associated with both the Roll Program and the Flight Instrument hidden states. Because HMM analysis takes into account the displays that were fixated both before and after the current display, fixations associated with one state or the other can be disambiguated.

Another advantage of the HMM approach is the capability for data soft-bordering. For instance, the F2-F3 (1) fixations were included in the Flight Instruments hidden state with relatively smaller weights, so that some data misclassified into this area adjacent to the HSI/SPD (2) and the ADI/ALT (3) could still be correctly identified as part of the Flight Instrument hidden state. This soft-bordering capacity of HMM analysis gives the analysis robustness and is expected to be increasingly helpful in future research on more complex electronic multifunctional cockpit displays. Such displays tend to present many pieces of information in one display region, and, consequently, defining borders between each data cluster associated with 
each piece of information will be more and more difficult. However, because of the soft-bordering capacity, HMM analysis can be more forgiving of potential data misclassifications.

The study also demonstrated the ability of HMM analysis to automatically detect deviations from regular scanning patterns. The computation can be run in real-time with a small delay. Thus, in addition to display studies, HMM analysis also may provide interesting applications for astronaut training. For example, instructors may be able to tell when their trainees interrupted regular scanning of the instruments. While this paper used a 15 -second delay, shorter delays also could be used if desired.

In addition to the various advantages, the study also revealed limitations of any analysis based purely on eye movements. Any analysis (including HMM) that relies on the existence of patterns in eye-movement data cannot identify hidden states that do not yield unique, detectable patterns (such as the hidden states associated with the flash evaporator check or the roll-to-heads-up check). While this may seem obvious and mostly a philosophical issue, it is important to note that behavior-based techniques cannot distinguish underlying cognitive states that produce identical behaviors. Thus, it is possible that some crewmembers may have additional hidden states that cannot be directly identified from their eye-movement data because the associated scan patterns are too similar to those associated with other hidden states. HMM analysis is still useful, but care should be taken in interpreting the results.

Nonetheless, HMM analysis still appears to have the potential to provide advantages over conventional scanning behavior studies, which typically rely on simple statistical analyses of raw eye-movement data. HMM analysis can supplement these eye-movement data statistics with the additional information provided by the hidden-state process, which is more directly related to the crewmembers' cognitive process. The organizations of the resulting HMM structures themselves also provide useful information about the basic scanning strategies of the crewmembers. These additional measures provided by HMM analysis can be useful in the future investigation of the effects of various proposed cockpit display designs for the next-generation spacecraft or for relating the various skill levels of the crewmembers to their scanning behavior.

\section{Conclusion}

The paper has described a novel HMM analysis to investigate the scanning behavior of Space Shuttle crewmembers. A proof-of-concept demonstration identified several potential advantages of using HMM analysis for human-centered cockpit interface design or for evaluating individual crewmembers' skill levels based on their scan patterns. First, the flexibility of HMM analysis allows analysis of complex cases, in which a display or displays overlap among multiple hidden states. Second, the robustness of HMM analysis also can alleviate the requirement of enforcing exact borders between areas associated with different pieces of information. Third, the estimated HMM model parameters can be used to detect deviations from crewmembers' regular scan patterns. This technique could be useful in astronaut training programs. Fourth, the additional measures obtained by HMM analysis have the potential to provide further insight into crewmembers' scanning strategies.

\section{Acknowledgement}

This research was conducted as a part of the research project funded by the Space Human Factors Engineering Grant \#01-OBPR-07, the Engineering for Complex Systems Program (Resilient Systems and Operations), and the Shuttle Cockpit Avionics Upgrade Project. The authors express appreciation to the members at the ISIS lab, Valerie Huemer, Fritz Renema, Steve Elkins, Bob Lawrence, and Jeff McCandless, at NASA Ames Research Center for their assistance and useful feedback. The authors are also extremely grateful to the astronaut who participated in this study.

\section{References}

[1] M. Hayashi, "Hidden Markov Models to Identify Pilot Instrument Scanning and Attention Patterns," in Proc. of the IEEE International Conference on Systems, Man, and Cybernetics, Washington DC, 2003, pp. 2889-2896.

[2] W. F. Clement, H. R. Jex, and D. Graham, "Manual control-display theory applied to instrument landings of jet transport," IEEE Transactions on Man-Machine Systems, vol. MMS-9, pp. 93-110, 1968.

[3] USA, "Shuttle Crew Operations Manual: OI-29," United Space Alliance, Houston, TX SFOC-FL0884, Rev. B., CPN-2, May 102002.

[4] L. Rabiner and B.-H. Juang, Fundamentals of Speech Recognition. Englewood Cliffs, NJ: Prentice Hall, 1993. 


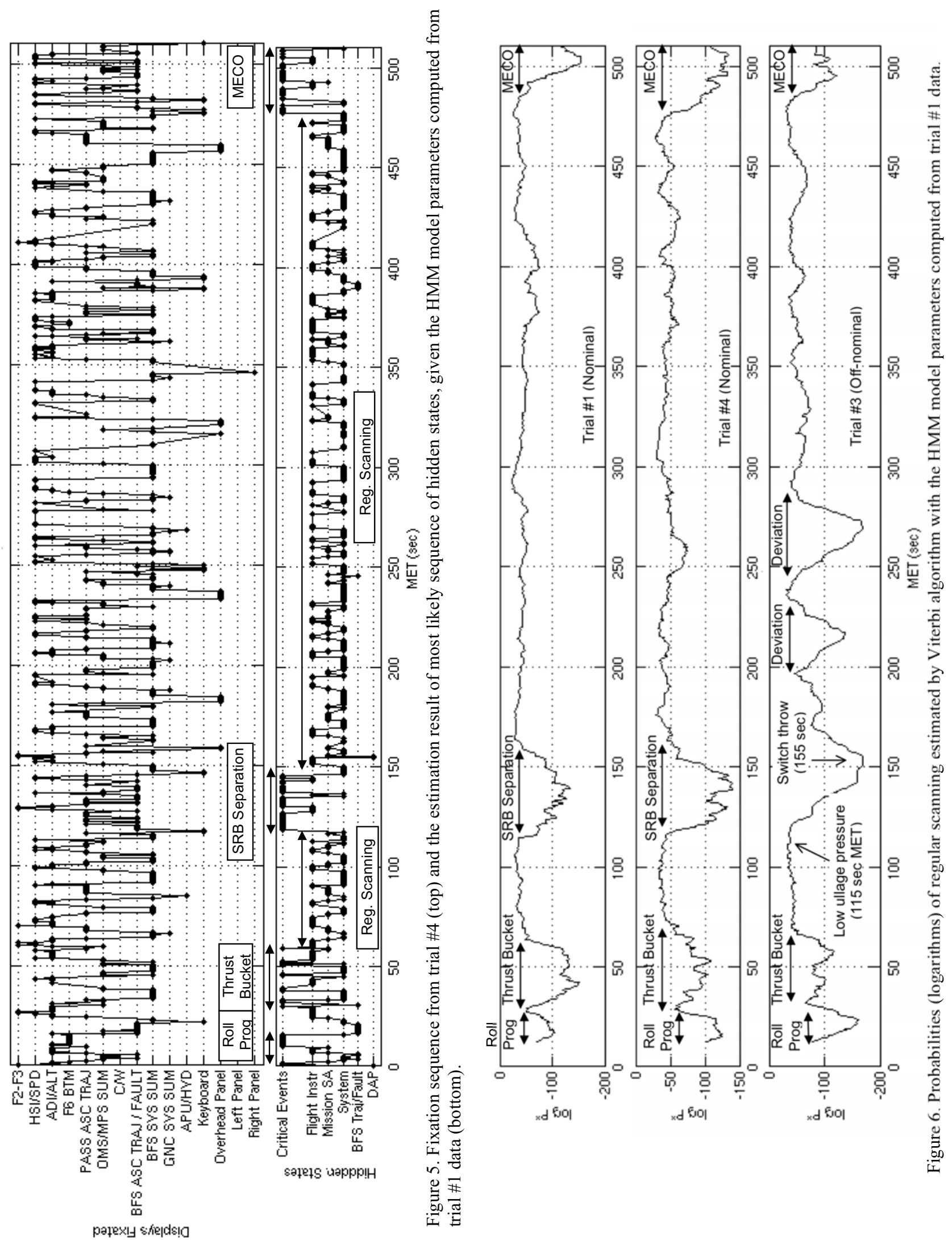

